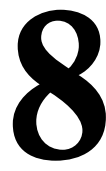

\title{
PERCEPCIONES SOBRE EL USO DE RECURSOS TIC Y «MASS-MEDIA» PARA LA ENSEÑANZA DE LA HISTORIA. UN ESTUDIO COMPARATIVO EN FUTUROS DOCENTES DE ESPAÑA-INGLATERRA ${ }^{1}$
}

(PERCEPTIONS ON THE USE OF ICT RESOURCES AND «MASS-MEDIA» FOR THE TEACHING OF HISTORY. A COMPARATIVE STUDY AMONG FUTURE TEACHERS OF SPAIN-ENGLAND)

Pedro Miralles Martínez

Cosme J. Gómez Carrasco

José Monteagudo Fernández

Universidad de Murcia

DOI: 10.5944/educXX1.21377

Cómo referenciar este artículo/How to reference this article:

Miralles Martínez, P.; Gómez Carrasco, C.J. y Monteagudo Fernández, J. (2019). Percepciones sobre el uso de recursos TIC y «mass-media» para la enseñanza de la historia. Un estudio comparativo en futuros docentes de España-Inglaterra. Educación XX1, 22(2), 187-211, doi: 10.5944/educXX1.21377

Miralles Martínez, P.; Gómez Carrasco, C.J. \& Monteagudo Fernández, J. (2019). Perceptions on the use of ICT resources and mass-media for the teaching of History. A comparative study among future teachers of Spain-England. Educación XX1, 22(2), 187-211, doi: 10.5944/ educXX1.21377

\section{RESUMEN}

El uso de las TIC y los «mass-media» se ha convertido en una de las principales líneas de investigación sobre el proceso de enseñanzaaprendizaje y la innovación educativa. El objetivo de ese artículo es conocer la opinión del futuro profesorado de ciencias sociales en España e Inglaterra sobre el empleo de diferentes recursos para la enseñanza de la historia en Educación Secundaria. Para ello se ha llevado a cabo una investigación de tipo cuantitativo no experimental a través de un cuestionario con una escala de valoración cerrada tipo Likert (1-5) en 22 universidades de España e Inglaterra con una amplia representación territorial, y con una muestra de 
504 cuestionarios. Se han detallado los resultados descriptivos de uno de los ítems del cuestionario relacionado con el objetivo de este trabajo, y se ha realizado la descripción de algunos de los factores a través del análisis de componentes principales con rotación varimax. Los datos extraídos muestran algunas diferencias significativas entre el futuro profesorado español e inglés en lo que al uso de las TIC y los «mass-media» se refiere. Unas diferencias que muestran una percepción sobre el uso de estos recursos más motivacional en el caso español y más procedimental en el caso inglés. Se hace necesario mejorar la formación docente en España, tanto inicial como permanente, en vista a conseguir unas clases de historia más dinámicas a través del uso de las TIC y los «mass-media» como recursos que permiten replicar el trabajo del historiador en clase, mejorar las actividades de tipo experiencial en la formación histórica del alumnado, y no meramente como un elemento lúdico.

\section{PALABRAS CLAVE}

Formación del profesorado; enseñanza de la historia; educación secundaria; TIC; medios de comunicación de masas.

\section{ABSTRACT}

The use of ICT and mass media has become one of the main lines of research for the teaching-learning process and educational innovation. The aim of this paper is to learn the opinion of future teachers of Social Sciences in Spain and England on the use of different resources for the teaching of History in Secondary Education. To this end, non-experimental quantitative research was carried out through a questionnaire with a closed Likert rating scale (1-5) in 22 universities in Spain and England with a broad territorial representation, and with a sample of 504 questionnaires. The descriptive results of one of the items of the questionnaire related to the objective of this work have been detailed, and a description of some of the factors has been made through the analysis of principal components with Varimax rotation. The data extracted shows some significant differences between the future Spanish and English teachers regarding the use of ICT and mass media. Some differences show a more motivational perception of the use of these resources in the Spanish case, which is more procedural in England. It is necessary to improve teacher training in Spain, both initial and permanent, in order to achieve more dynamic History classes through the use of ICT and mass media as resources that allow the work of the historian to be replicated in class, to improve experiential activities in the historical formation of the students, and not merely as a playful element. 


\section{KEYWORDS}

Teacher training; History education; secondary education; ICT; mass media.

\section{INTRODUCCIÓN}

Las nuevas tecnologías de la información y la comunicación (TIC), así como los medios de comunicación de masas («mass-media»), ocupan un papel muy importante en la vida diaria. Como sostenía Marqués (2013), «están ahí, forman parte de la cultura tecnológica que nos rodea y con la que debemos convivir» (p. 2). Por ello compartimos la opinión de Ramírez y González (2016) de que las nuevas investigaciones sobre dichos fenómenos ya no han de centrarse en su influencia en la sociedad, sino sobre qué se hace con ellos, especialmente en el ámbito educativo, donde una de las principales funcionalidades de las TIC y los mass-media es su uso didáctico para facilitar los procesos de enseñanza y aprendizaje (Marqués, 2013).

Aunque el profesorado y el alumnado viven inmersos en experiencias mediáticas, la transferencia al proceso de enseñanza y aprendizaje no se aprecia realmente en la mayoría de los centros escolares debido a las reservas del propio profesorado hacia el uso de los TIC y los mass-media (Ramírez y González, 2016), reservas relacionadas con su formación, la variable que más incide en el nivel de competencia mediática (González, Gozálvez y Ramírez, 2015). Ello hace que en la mayoría de ocasiones la labor del enseñante se quede, siguiendo a Marqués (2013), en un escenario de aprender DE las TIC y no se aprenda CON las TIC, para así poder innovar en las prácticas docentes e introducir cambios reales en las aulas que lleven a conseguir mejores aprendizajes (De Haro, 2009).

Ya defendía Bates (2009, citado por Romero, Castejón, López y Fraile, 2017, p. 75) que la simple presencia de nuevas tecnologías en los centros de enseñanza no garantiza la innovación, sino que son necesarios cambios en el rol del profesor y de los estudiantes, en la metodología y en el sistema de evaluación (Marqués, 2013; Romero et al., 2017).

Además, la evolución que sufren las propias TIC hace que el uso de las mismas deba estar relacionado con las herramientas de la Web 2.0 y la puesta en marcha de metodologías de aprendizaje colaborativo si verdaderamente se apuesta, como ya se ha apuntado, por procesos de innovación educativa.

Respecto a los «mass-media», Cabero y Guerra (2011) sostienen que una persona formada y alfabetizada en medios adquiere una serie 
de competencias que le ayudarán a ser autónoma, crítica, reflexiva y participativa en la sociedad de la información en la que vivimos actualmente. Del mismo modo, para Ortega y Pérez (2013) la alfabetización mediática y visual es imprescindible en las sociedades avanzadas del conocimiento y de la era digital dado que un tercio de la información que llega a la ciudadanía es de naturaleza visual, así que el uso de la prensa, la fotografía o el cómic en el aula es necesario.

Todo lo hasta ahora expuesto presenta un cariz más ineludible en un campo de conocimiento como el de las ciencias sociales, cuya principal función es la formación de ciudadanía.

En la última década los estudios sobre la enseñanza de las ciencias sociales, como en otras didácticas aplicadas, han insistido en la necesidad de incluir las nuevas tecnologías de la información y de la comunicación en el aula (Miralles, Gómez y Arias, 2013). Si el propósito de la didáctica de las ciencias sociales según Hernàndez Cardona (2011) es analizar, diseñar e investigar acerca de las técnicas didácticas, de divulgación y comunicación de los contenidos de geografía, historia e historia del arte, así como los procesos de comprensión de los mismos, las nuevas tecnologías suponen una oportunidad para el docente como generador de imágenes virtuales en el espacio y el tiempo. El uso de estos recursos aumenta las opciones de experimentación en las ciencias sociales (Martín, 2011). Como opina Acosta (2010), la historia se encuentra en desventaja respecto a las ciencias experimentales, incluso con otras ciencias sociales, debido a la imposibilidad de reproducir los hechos históricos objeto de estudio. La introducción de las TIC da la oportunidad a los docentes de solventar algunos de estos problemas. Es decir, es una opinión bastante generalizada que la utilización de las TIC y el camino hacia un aula virtual es un valioso recurso en el aprendizaje de las ciencias sociales en todos los niveles educativos (Osset, 2004).

Las principales ventajas esgrimidas sobre el uso de las nuevas tecnologías para la enseñanza de la geografía, historia y otras ciencias sociales han girado en torno al aprendizaje autorregulado y significativo que permiten las TIC, la colaboración, tanto entre alumnos, como entre docentes y estudiantes, y el hecho de que la enseñanza pueda ser más individualizada. El uso de blog, wiki, webquest, cazatesoros, podcast, videojuegos, dispositivos móviles, realidad aumentada, pizarra digital interactiva, redes sociales, etc. están ya presentes en la enseñanza de la historia más innovadora. Hernàndez Cardona (2011) o Rivero (2011) han mostrado que la utilización de imágenes virtuales, la interactividad de las nuevas tecnologías o el uso de la multimedia expositiva permiten potenciar eficazmente la educación histórica. Algunas autoras como la propia Rivero (2010), destacan que las nuevas herramientas didácticas multimedia facilitan el mayor acceso a 
fuentes primarias que permiten la construcción del conocimiento histórico. La arqueología virtual, por ejemplo, es una herramienta que puede ayudar a explicar la evolución de los paisajes culturales a través de las recreaciones 3D, llevando a cabo una actividad amena y participativa (López-Menchero y Ramiro, 2015). El uso de blogs en el aula de historia es otro de los principales motores de innovación que se ha implantado principalmente en Educación Secundaria (Sobrino, 2013). Y relacionado con el uso de las TIC también está la introducción de los videojuegos para la enseñanza de la historia como recurso educativo (Cuenca, Martín y Estepa, 2011; Iturriaga, 2015; Rodríguez y Gutiérrez, 2016).

También en este sentido se encuentra la línea de investigación desarrollada por investigadores del área de Didáctica de las Ciencias Sociales de la Universidad del País Vasco sobre el uso de las nuevas tecnologías para la enseñanza y difusión del patrimonio en el ámbito escolar (Ibáñez, Vincent y Asensio, 2012; Ibáñez, Vicent, Asensio, Cuenca y Fontal, 2014; Vincent, Ibáñez y Asensio, 2015). Esta línea de investigación apuesta por la tecnología educativa Lurquest fundamentada en las teorías del aprendizaje contextual y de la integración significativa de la tecnología móvil en el proceso de enseñanza-aprendizaje, con el objetivo de evolucionar y complementar los conceptos WebQuest, geocatching y museo territorio, llegando a implementar un programa de aprendizaje de patrimonio y arqueología a través de tecnología m-learning (aprendizaje móvil) en un entorno físico concreto. El trabajo de Colomer (2016) sigue esa línea.

La realidad aumentada es otra de las tecnologías emergentes que se está experimentando en los últimos años para la enseñanza de las ciencias sociales. No son muchas todavía las publicaciones sobre este recurso, pero algunas ya van avanzando en sus efectos positivos. Los trabajos de Cózar, De Moya, Hernández y Hernández (2015), de López y Miralles (2018) y de Sáez y Cózar (2017), tanto en la formación de profesorado de Educación Primaria y Secundaria como en el aula de Ciencias Sociales en Primaria, nos ponen ejemplos de experiencias evaluadas con resultados positivos. El aumento de la motivación, la atención, los planteamientos activos, el incremento del interés, el pensamiento crítico o las actividades colaborativas son expuestos como los principales argumentos para la introducción de este recurso.

La gamificación como estrategia metodológica para la enseñanza de las ciencias sociales tiene una bibliografía relativamente notable en los últimos 25 años. Si nos fijamos en las propuestas metodológicas, los ejemplos que se han publicado últimamente nos muestran un avance significativo para trabajar en el aula de ciencias sociales. Entre ellos el trabajo de Ayén (2017), explicando toda la dinámica metodológica y los procedimientos para 
establecer puntos ranking, etc.; Guevara y Colomer (2017) con propuestas para Primaria y Secundaria con Eduloc y Minecraft; Cózar y Sáez (2016) con experiencias evaluadas con el uso de Minecraft; Sáez y Cózar (2017) con experiencias en Primaria utilizando la programación visual por bloques; o Maté (2017) con pautas para el uso de juegos de rol para favorecer cambios actitudinales y facilitar el aprendizaje por experiencia. En todos estos trabajos se han resaltado los resultados positivos en los dos aspectos indicados anteriormente: la motivación del alumnado, que lo predispone para el aprendizaje. Y el aprendizaje experiencial, que hace que la asimilación de los conocimientos de ciencias sociales sea más significativa.

Dentro de las propuestas gamificadoras, el uso de videojuegos está teniendo una importante bibliografía. Propuestas y experiencias desde una perspectiva general del aprendizaje como las de Contreras, Eguía y Solano (2011), Del Moral (2012) o Gros (2008) han resaltado los efectos positivos que tiene la introducción de este recurso. Los videojuegos pueden clasificarse por el dispositivo en el que se ejecutarán, por temáticas, por el papel que juega el jugador, etc. Lo más importante cuando utilizamos el videojuego en el aula no es el resultado sino el proceso que se ha seguido para enfrentarse a los problemas y resolverlos mediante una toma de decisiones adecuada y sistemática. Esto favorece al aprendizaje del alumno porque el objetivo es obtener el conocimiento mediante la compresión de las características del problema.

La publicación de propuestas y experiencias con el uso de los videojuegos para la enseñanza de las ciencias sociales se ha incrementado de forma exponencial en los últimos seis-siete años. Trabajos como los de Cuenca, Martín y Estepa (2011) fueron en cierta manera pioneros, y mostraron el potencial didáctico de este recurso. En los últimos años, las publicaciones de Iturriaga y Medel (2017), Jiménez y Cuenca (2015 y 2017), Jiménez y Rodríguez (2015), Martínez (2017), Téllez e Iturriaga (2014) o Rodríguez y Gutiérrez (2016), han profundizado en estos aspectos. De nuevo, la motivación del alumnado junto con el trabajo experiencial han sido los principales argumentos expuestos para mostrar los efectos positivos del uso de este recurso en el aula de ciencias sociales.

\section{METODOLOGÍA}

\section{Objetivos}

El objetivo principal de este trabajo es analizar las opiniones de los futuros docentes de Educación Secundaria sobre el uso de las TIC y los mass-media para la enseñanza de la historia en relación a otro tipo de 
recursos y materiales. Para conseguir este objetivo se han planteado una serie de objetivos específicos:

1. Describir y diferenciar la valoración que los futuros docentes de Secundaria otorgan a diferentes recursos para la enseñanza de la historia.

2. Analizar las valoraciones de los futuros docentes de España e Inglaterra sobre el uso de las TIC y los mass-media para la enseñanza de la historia.

\section{Diseño de la investigación e instrumento de recogida de información}

El diseño empleado en la presente investigación ha sido un diseño cuantitativo no experimental tipo encuesta. Se ha optado por este tipo de diseño debido a que se trata de un método de investigación capaz de dar respuesta a problemas tanto en términos descriptivos como de relación de variables cuando la información es recogida de forma sistemática, garantizando el rigor de los datos obtenidos (Buendía, Colás y Hernández, 1998; Hernández y Maquilón, 2010).

Los datos utilizados para este trabajo forman parte de un cuestionario con una escala de valoración cerrada tipo Likert (1-5). El cuestionario fue titulado «Opinión y percepción del profesorado en formación inicial sobre el aprendizaje de la historia y la evaluación de competencias históricas». El cuestionario tiene una parte de identificación y tres bloques temáticos. El primer bloque, titulado «Opinión y percepción sobre la evaluación y su papel en el proceso de enseñanza y aprendizaje», contiene 12 afirmaciones sobre el papel de la evaluación que los participantes debían valorar desde 1 (totalmente en desacuerdo) hasta 5 (totalmente de acuerdo). El segundo bloque, titulado «Opinión y percepción sobre la historia como materia formativa, métodos, fuentes y recursos de enseñanza», contiene 15 afirmaciones que los participantes deben valorar desde 1 (totalmente en desacuerdo) hasta 5 (totalmente de acuerdo), y 24 métodos, técnicas y recursos didácticos para la enseñanza de la historia que los participantes deben valorar desde 1 (menos relevante) hasta 5 (más relevante). El tercer bloque, titulado «Opinión y percepción sobre la evaluación de competencias históricas en Educación Secundaria: utilización de fuentes, razonamiento causal y empatía histórica», contiene 9 afirmaciones que los participantes deben valorar desde 1 (totalmente en desacuerdo) hasta 5 (totalmente de acuerdo), y 8 actividades de evaluación de competencias históricas que los participantes deben valorar desde 1 (menos relevante) hasta 5 (más relevante). Los ítems del cuestionario se han construido teniendo en cuenta las prácticas docentes relacionadas con perfiles tradicionales e innovadores (Gómez, Miralles y Chapman, 2017). 
El cuestionario fue validado por cuatro expertos, tres de ellos del área de Didáctica de las Ciencias Sociales de tres universidades distintas, y con amplia experiencia en Educación Secundaria. El cuarto validador fue profesor del área de Métodos de Investigación y Diagnóstico en Educación. Los expertos validadores rellenaron un cuestionario con una escala Likert de 1-4. Se dejaron solo los ítems que superaran el tres de media por los validadores. Además se modificaron todas las cuestiones señaladas de forma cualitativa. Tras realizar la validación del cuestionario por los expertos, el cuestionario fue traducido al inglés y se sometió a la validación del comité de ética del Institute of Education (University College of London) para poder pasarlo en centros ingleses. Para ello se rellenó el «Staff Ethics Application Form» con la información sobre los procedimientos y la metodología que se iba a utilizar en la recogida y el análisis de la información. El comité de ética del Institute of Education resolvió positivamente sobre el cuestionario, lo que permitió distribuirlo en centros españoles e ingleses entre mayo y junio de 2016 (Miralles, Gómez y Rodríguez, 2017).

\section{Tabla 1}

Ítem 2.24 del cuestionario. Valora del 1 (menos relevante) al 5 (más relevante) los materiales y recursos que según su opinión son más adecuados para la enseñanza de la historia en Educación Secundaria

2.24.1. Libro de texto

2.24.2. Internet

2.24.3. Fuentes documentales primarias

2.24.4. Fuentes orales (entrevistas a abuelos, familiares, vecinos...)

2.24.5. Prensa diaria impresa o digital

2.24.6. Apuntes del profesor

2.24.7. Museos y otros centros de interés histórico y patrimonial

2.24.8. Cine histórico

2.24.9. Documentales de temática histórica

2.24.10. Novela histórica

2.24.11. Revistas de divulgación

2.24.12. Videojuegos

2.24.13. Cómics

2.24.14. Fiestas y tradiciones locales de contenido histórico

2.24.15. Otra (indica cuál): 
Para este trabajo se ha escogido el ítem 2.24 (Tabla 1) dentro del bloque 2 del cuestionario. En este ítem se pedía a los futuros docentes que valoraran del 1 (menos relevante) al 5 (más relevante) los materiales y recursos que en su opinión consideraba más adecuados para la enseñanza de la historia en Educación Secundaria.

Para analizar la validez de constructo del cuestionario se ha realizado un análisis de la estructura factorial por medio del análisis de componentes principales con rotación Varimax y valores Eigen mayor que uno de los diferentes bloques de ítems. Antes de realizar el proceso de factorización se ha determinado el grado de adecuación de los datos obtenidos mediante el índice de adecuación muestral de Kaiser-Meyer-Olkin (KMO) que oscila entre cero y uno, indicando que el análisis factorial es adecuado cuanto mayor sea su valor, no resultando pertinente realizarlo con valores <.60. También se calculó el test de esfericidad de Bartlett, así como la diagonal de la matriz de correlaciones anti-imagen. El índice de adecuación muestral KMO del bloque 2 del cuestionario (donde se inserta el ítem 2.24) resultó ser .763 en caso de la muestra de universidades españolas (tabla 2) y de .645 en caso de la muestra de universidades inglesas (tabla 3). La prueba de esfericidad de Barlett resultó significativa en ambos casos, lo cual indica que la matriz es adecuada para ser factorizada. Con estos datos, procedimos a la realización del análisis factorial obteniendo 10 factores que explican los 39 ítems del segundo bloque.

Tabla 2

KMO y prueba de Bartlett de la factorización del bloque II del cuestionario en España

\section{KMO y prueba de Bartlett}

Medida de adecuación muestral de Kaiser-Meyer-Olkin. ,763

Chi-cuadrado aproximado 2961,488

Prueba de esfericidad de Bartlett gl

Sig.

, 000

\section{Tabla 3}

KMO y prueba de Bartlett de la factorización del bloque II del cuestionario en Inglaterra

\section{KMO y prueba de Bartlett}

Medida de adecuación muestral de Kaiser-Meyer-Olkin. , 645

Chi-cuadrado aproximado

2233,232

Prueba de esfericidad de Bartlett gl 


\section{Participantes}

En la investigación participaron 506 docentes en formación que cursaban el Máster de Formación del Profesorado de Educación Secundaria en la especialidad de Geografía e Historia en España (344) y del Postgraduate Certificate in Education (PGCE) y el Teach First en Inglaterra (162) al finalizar el curso académico 2015/2016. En la investigación participaron 22 universidades, 13 españolas y 9 inglesas. Se priorizó tener una amplia representación territorial.

En el caso español, se contactó con los coordinadores y el profesorado del Máster de Formación del Profesorado de la especialidad de Geografía e Historia. Se enviaron los cuestionarios en papel a las universidades que respondieron favorablemente a esta petición lo largo del mes de mayo de 2016. En el caso inglés, tras contactar en mayo previamente con el profesorado encargado de la materia, unas universidades optaron por recibir los cuestionarios en papel, y otros profesores prefirieron el envío de un link con el cuestionario en formato electrónico y ellos distribuirlo entre el alumnado. Así, recogimos los cuestionarios en papel del IoE-UCL, University of Exeter y University of Edge Hill. Mientras que recibimos la respuesta a los cuestionarios on-line de docentes en formación de la Metropolitan Manchester University, University of York, University of Leeds, East-Anglia University, University of Birmingham, y de la Christ Church University of Cantebury $^{2}$. Los datos demográficos de la muestra están presentes en las tablas 4,5 y 6 .

Tabla 4

Participantes que respondieron al cuestionario en España e Inglaterra

\begin{tabular}{lcc}
\hline & Frecuencia & Porcentaje \\
\hline España & 344 & 67,9 \\
Inglaterra & 162 & 32,1 \\
\hline Total & 506 & 100,0 \\
\hline
\end{tabular}




\section{Tabla 5}

Edad de los participantes que respondieron al cuestionario en España e Inglaterra

\begin{tabular}{cccccc}
\hline & & $\begin{array}{c}\text { Frecuencia } \\
\text { España }\end{array}$ & $\begin{array}{c}\text { Porcentaje } \\
\text { España }\end{array}$ & $\begin{array}{c}\text { Frecuencia } \\
\text { Inglaterra }\end{array}$ & $\begin{array}{c}\text { Porcentaje } \\
\text { Inglaterra }\end{array}$ \\
\hline \multirow{6}{*}{ NTota } & $20-25$ & 214 & 62,2 & 98 & 60,5 \\
& $26-30$ & 68 & 19,8 & 33 & 20,4 \\
& $31-35$ & 18 & 5,2 & 3 & 1,9 \\
& $36-40$ & 12 & 3,5 & 6 & 3,7 \\
& $41-45$ & 3 & 0,9 & 4 & 2,5 \\
& $46-50$ & 0 & 0,0 & 1 & 0,6 \\
& $51-55$ & 0 & 0,0 & 0 & 0,0 \\
& $56-60$ & 0 & 0,0 & 0 & 0,0 \\
& + de 60 & 1 & 0,3 & 0 & 0,0 \\
& NC & 28 & 8,1 & 17 & 10,5 \\
\cline { 2 - 6 } & Total & 344 & 100,0 & 162 & 60,5 \\
\hline
\end{tabular}

Tabla 6

Género de los participantes en el cuestionario en España e Inglaterra

\begin{tabular}{llccrc}
\hline & & $\begin{array}{c}\text { Frecuencia } \\
\text { España }\end{array}$ & $\begin{array}{c}\text { Porcentaje } \\
\text { España }\end{array}$ & $\begin{array}{c}\text { Frecuencia } \\
\text { Inglaterra }\end{array}$ & $\begin{array}{c}\text { Porcentaje } \\
\text { Inglaterra }\end{array}$ \\
\hline & Hombre & 187 & 54,4 & 61 & 37,7 \\
& Mujer & 139 & 40,4 & 100 & 61,7 \\
& Total & 326 & 94,8 & 161 & 99,4 \\
\hline Perdidos & Sistema & 18 & 5,2 & 1 &, 6 \\
\hline Total & & 344 & 100,0 & 162 & 100,0 \\
\hline
\end{tabular}

\section{ANÁLISIS Y RESULTADOS}

En la tabla 7 se muestran los estadísticos descriptivos del bloque 2.24 del cuestionario, en el que los docentes en formación debían valorar diferentes recursos por su adecuación a la enseñanza de la Historia en Educación Secundaria. Como se puede comprobar en dicha tabla, el ítem "Museos y otros centros de interés histórico» es el más valorado por los docentes en formación en España, y el segundo más valorado en el caso de los docentes en formación ingleses. En Inglaterra solo lo supera la valoración del ítem «Fuentes documentales primarias». En el caso de España, además del ítem de museos, solo superan el 4 de valoración las 
fuentes documentales primarias, las fuentes orales y documentales de temática histórica. En el caso de Inglaterra solo superan el 4 de valoración el ítem de museos y el de fuentes documentales primarias. Los ítems con menor apreciación en el caso de España son el libro de texto, los cómics y los videojuegos. En el caso inglés, los ítems de menor estimación son los cómics, los videojuegos y la novela histórica. Los ítems relacionados con las TIC y los mass-media (Internet, cine, documentales, revistas de divulgación, novela histórica, cómics y videojuegos) recibieron una puntación desigual, desde una valoración media-alta (Internet, cine y documentales), a una valoración baja (cómics y videojuegos).

Tabla 7

Estadísticos descriptivos de los ítems del bloque 2.24 del cuestionario

\begin{tabular}{lcccccc}
\hline & N. España & $\begin{array}{c}\text { Media } \\
\text { España }\end{array}$ & $\begin{array}{c}\text { Desviación } \\
\text { estándar } \\
\text { España }\end{array}$ & $\begin{array}{c}\text { N. } \\
\text { Inglaterra }\end{array}$ & $\begin{array}{c}\text { Media } \\
\text { Inglaterra }\end{array}$ & $\begin{array}{c}\text { Desviación } \\
\text { estándar } \\
\text { Inglaterra }\end{array}$ \\
\hline B2.24.1 & 343 & 2,97 & 1,042 & 162 & 3,39 &, 986 \\
B2.24.2 & 343 & 3,78 &, 895 & 162 & 3,63 &, 958 \\
B2.24.3 & 343 & 4,23 &, 815 & 162 & 4,59 &, 596 \\
B2.24.4 & 343 & 4,07 &, 871 & 162 & 3,86 &, 962 \\
B2.24.5 & 343 & 3,90 &, 915 & 162 & 3,62 &, 892 \\
B2.24.6 & 343 & 3,70 &, 952 & 162 & 3,53 &, 879 \\
B2.24.7 & 343 & 4,31 &, 822 & 162 & 4,23 &, 733 \\
B2.24.8 & 343 & 3,96 &, 894 & 162 & 3,42 & 1,014 \\
B2.24.9 & 343 & 4,01 &, 836 & 162 & 3,91 &, 810 \\
B2.24.10 & 343 & 3,28 & 1,100 & 162 & 3,11 & 1,028 \\
B2.24.11 & 343 & 3,36 &, 935 & 162 & 3,47 &, 934 \\
B2.24.12 & 343 & 3,17 & 1,101 & 162 & 1,93 & 1,016 \\
B2.24.13 & 343 & 3,17 & 1,109 & 162 & 2,18 & 1,034 \\
B2.24.14 & 343 & 3,52 & 1,062 & 162 & 3,42 & 1,038 \\
\hline
\end{tabular}

A continuación ofrecemos una serie de figuras en las que puede observarse la escala completa de valoración que recibieron cinco de estos recursos relacionados con las TIC y los mass-media. En lo que a la valoración de Internet respecta (Figura 1), se obtuvo un notable reconocimiento por parte del profesorado en formación, con una puntuación muy similar en ambos casos, aunque es ligeramente más elevado entre los futuros docentes españoles (3,78 frente al 3,63 de los futuros docentes ingleses). Una valoración positiva que en otros estudios también ha comprobado Gómez (2015 y 2016). 


\section{(1)}

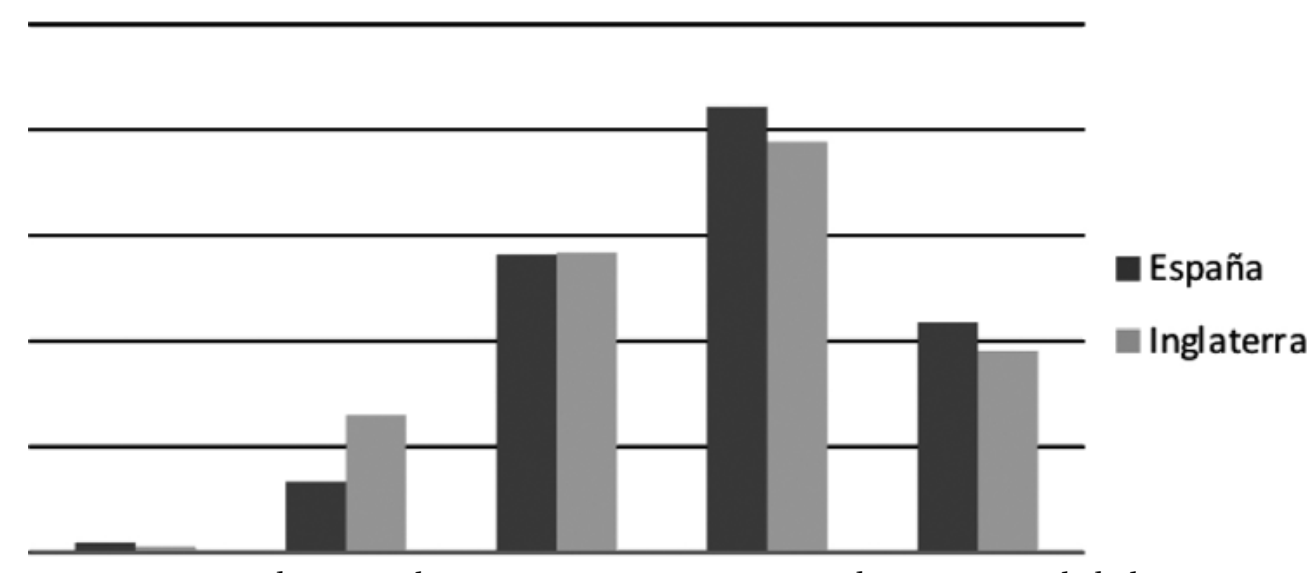

Figura 1. Valoración de Internet como recurso para la enseñanza de la historia en Educación Secundaria en futuros docentes de España e Inglaterra

La figura 2 muestra la valoración del cine histórico como recurso para la enseñanza de la Historia. Se trata de un recurso didáctico muy bien valorado debido a su capacidad para recrear el pasado o para reflejar el presente, lo que provoca fascinación en un alumnado predispuesto para los medios audiovisuales (Gorgues y Goberna, 1998; Sánchez, 1999). Nuevamente se observan unos resultados positivos para ambos casos, aunque vuelve a ser el profesorado en formación español quien le otorga una mejor valoración $(3,96)$, mientras que en el caso inglés desciende respecto a Internet $(3,42)$.

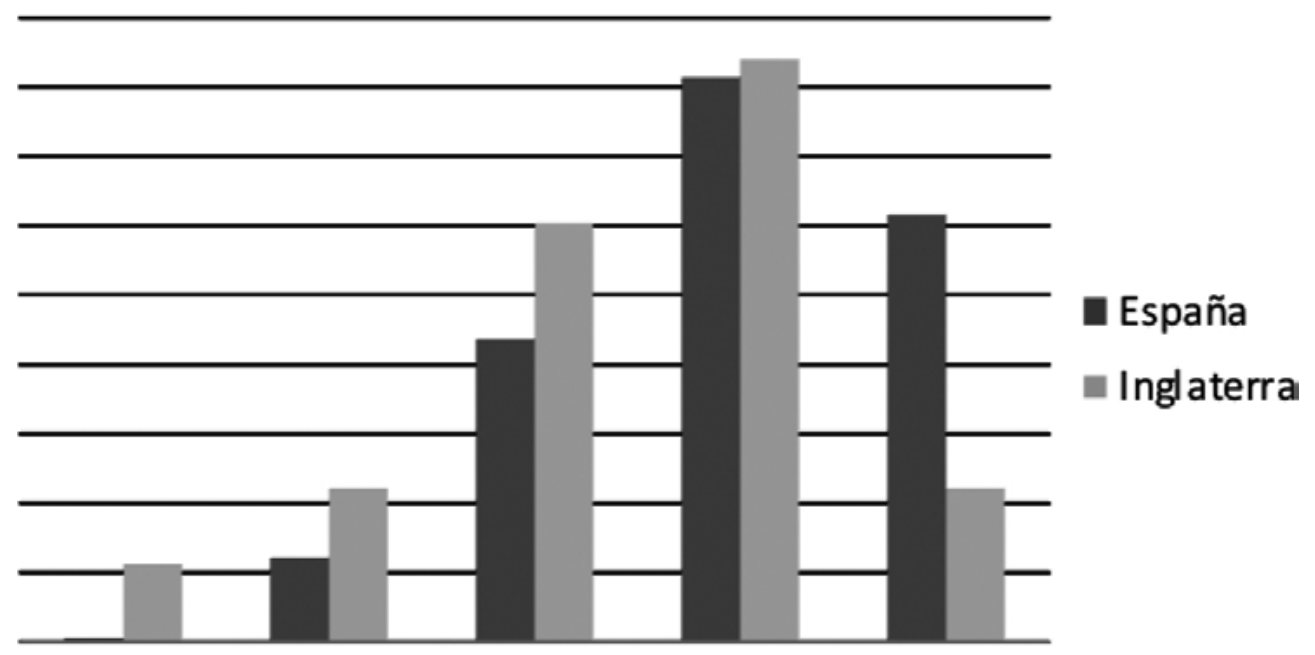

Figura 2. Valoración del cine como recurso para la enseñanza de la historia en Educación Secundaria en futuros docentes de España e Inglaterra 
La figura 3, referida los documentales de temática histórica, muestra una puntuación similar a la anterior. En el caso de España se sitúa en el cuarto puesto de todos los recursos valorado, con una media de 4 sobre 5 . Para los docentes ingleses en formación también es un recurso bien valorado, con una puntuación parecida al caso español $(3,91)$, y por encima sensiblemente del cine como recurso.

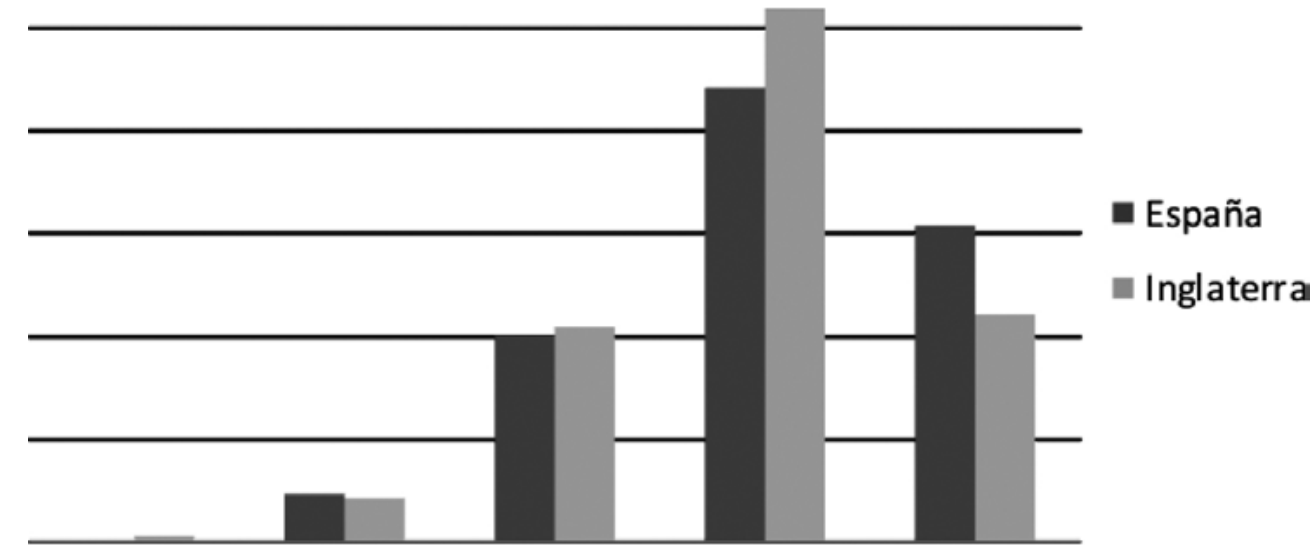

Figura 3. Valoración de los documentales de temática histórica como recurso de enseñanza de la historia en Educación Secundaria futuros docentes de España e Inglaterra

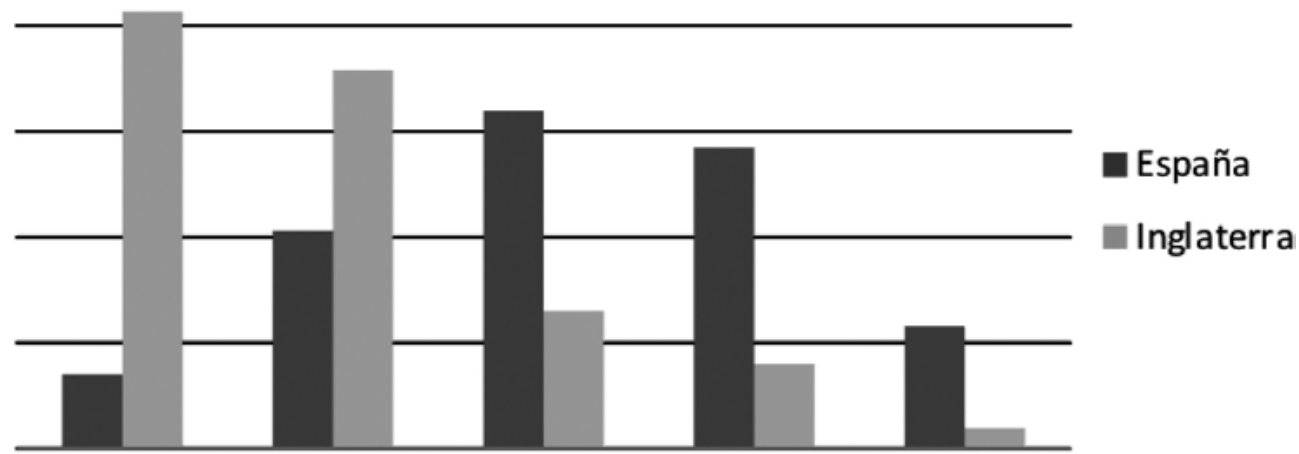

Figura 4. Valoración de los videojuegos como recurso de enseñanza de la historia en Educación Secundaria futuros docentes de España e Inglaterra

Más disparidad entre los resultados de España e Inglaterra se observa en la valoración recibida por los videojuegos (Figura 4) y los cómics (Figura 5). Aunque en España son de los recursos con menos puntuación, 
sigue estando valorado por encima de 3 sobre 5 en ambos casos. Sin embargo el profesorado inglés en formación valora ambos recursos de forma muy negativa, 1,93 sobre 5, en el caso de los videojuegos 2,18 sobre 5 en el caso de los cómics.

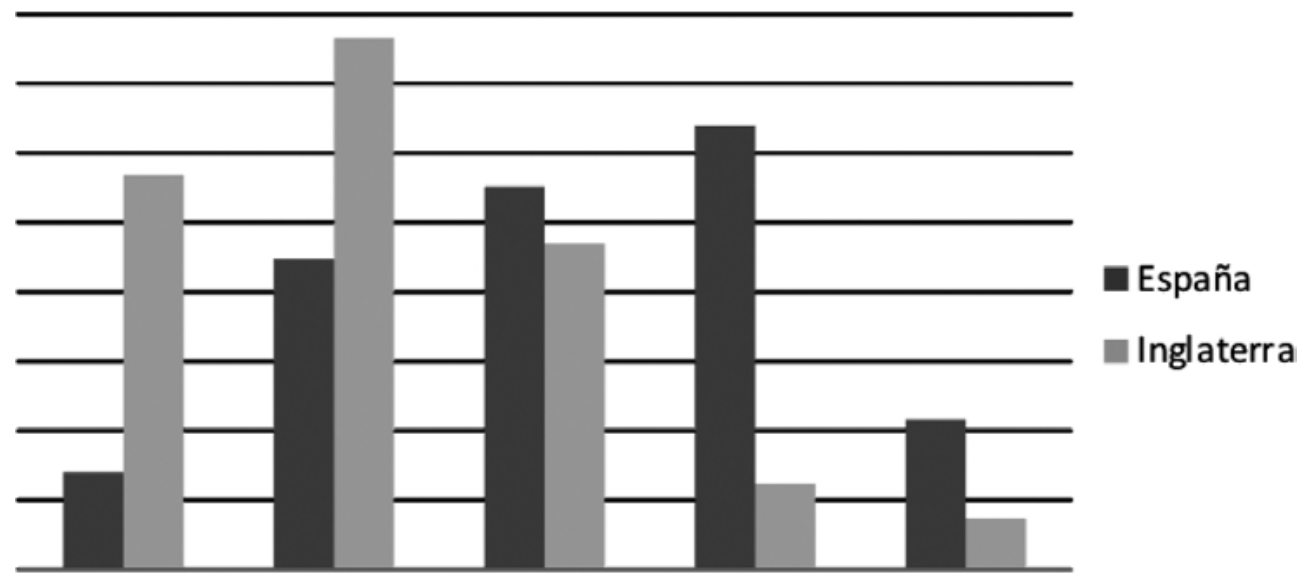

Figura 5. Valoración del cómic como recurso de enseñanza de la historia en Educación Secundaria futuros docentes de España e Inglaterra

Si además observamos la tabla 8 , con los resultados obtenidos de la factorización del bloque 2 del cuestionario, podemos comprobar cómo se agrupan los ítems sobre recursos TIC y mass-media en dos factores distintos. Tanto en España como en Inglaterra la valoración realizada a los cómics y videojuegos saturan en un factor con cargas muy similares (ítems B.2.24.13 y B.2.24.14), mientras que la novela histórica, los documentales, el cine y las revistas de divulgación saturan en un factor distinto también con cargas casi idénticas (ítems B2.24.8, B2.24.9, B2.24.10 y B2.24.11). Esto muestra cómo los docentes en formación españoles e ingleses perciben estos grupos de recursos de forma diferente. 
Tabla 8

Factores que interrelacionan ítems sobre TIC y mass-media dentro del Bloque 2 del cuestionario

\begin{tabular}{|c|c|c|c|c|}
\hline & España & Inglaterra & España & Inglaterra \\
\hline & Factor 1 & Factor 1 & Factor 2 & Factor 2 \\
\hline \multicolumn{5}{|l|}{ B2.24.1 } \\
\hline B2.24.2 & & & & ,442 \\
\hline \multicolumn{5}{|l|}{ B2.24.3 } \\
\hline \multicolumn{5}{|l|}{ B2.24.4 } \\
\hline B2.24.5 & & & & ,583 \\
\hline \multicolumn{5}{|l|}{ B2.24.6 } \\
\hline B2.24.7 & & & ,345 & ,437 \\
\hline B2.24.8 & & ,430 & ,738 & 638 \\
\hline B2.24.9 & & & ,787 & ,752 \\
\hline B2.24.10 & 379 & & 604 & ,704 \\
\hline B2.24.11 & & & ,499 & ,751 \\
\hline B2.24.12 & ,822 & ,812 & & \\
\hline B2.24.13 & ,807 & ,783 & & \\
\hline B2.24.14 & ,479 & & & \\
\hline
\end{tabular}

Método de extracción: Análisis de componentes principales.

Método de rotación: Normalización Varimax con Kaiser.

\section{DISCUSIÓN Y CONCLUSIONES}

Una vez expuestos los resultados de nuestro estudio, podemos establecer una serie de conclusiones sobre las valoraciones que el profesorado en formación realiza sobre el uso de las TIC y los massmedia para la enseñanza de la historia. En primer lugar, los docentes españoles e ingleses en formación otorgan una gran valoración a las fuentes documentales primarias, los museos y las fuentes orales como recursos en el aula de Historia. Una circunstancia que se da sobre todo en el caso inglés, donde la mayor valoración recae en las fuentes que permiten desarrollar el método del historiador. Esta mayor relevancia que los profesores en formación ingleses otorgan al uso de fuentes primarias sobre el resto de recursos alude a una diferente tradición docente, con una visión más metodológica y procedimental. Un diferente enfoque en el que han tenido un notable impacto proyectos de investigación como el Concepts History and 
Teaching Approaches (CHATA) o los cambios introducidos por el National Curriculum de 1991 (Cooper y Chapman, 2009). El proyecto CHATA, que continuó durante los primeros años de este siglo se basó en la adquisición de los conceptos de segundo orden por el alumnado y en el establecimiento de niveles de progresión de este conocimiento en edades comprendidas entre siete y catorce años (Domínguez, 2015). Por ello son interesantes las aportaciones realizadas desde los años ochenta por un gran número de autores (Lee, 2005; Lee y Ashby, 2000; Lee, Dickinson y Ashby, 2004) quienes abogaron por una enseñanza de la historia en la que se incluyeran de manera equilibrada tanto contenidos conceptuales como procedimentales, a los que comenzaron a denominar conceptos de segundo orden (MartínezHita y Gómez, 2018).

En España las fuentes documentales primarias comparten protagonismo con otros recursos como los museos (primer ítem en valoración en el caso español) y los documentales de temática histórica (más valorado que en el caso inglés). Además todos los recursos relacionados con los mass-media (novela histórica, revistas de divulgación, cine histórico, etc.) tienen una mayor valoración en los docentes españoles en formación. Interpretamos con estos resultados que los futuros docentes en España valoran en mayor medida el factor motivacional para enseñar Historia que los docentes en formación ingleses. Como se desprende de diversos estudios, existe todavía en España una hegemonía de la clase de historia tradicional expositiva y aburrida. Martínez, Souto y Beltrán (2006) y Martínez, Miralles y Navarro (2009) estudiaron los cuestionarios respondidos por más de 1.500 estudiantes universitarios, que habían estudiado Historia en institutos de la Región de Murcia, tanto BUP y COU como LOGSE, entre 1991 y 2002. Entre los recuerdos de las malas prácticas, citaban el predominio del libro de texto y la lección magistral, así como la ausencia casi total de las TIC en el aula. Los resultados obtenidos por Cuesta (1998), Gómez, Rodríguez y Mirete (2018) y Merchán (2007) fueron muy similares en tres realidades geográficas distintas (Salamanca, Murcia y Sevilla) y en tres décadas diferentes. Por los resultados obtenidos del cuestionario, los docentes en formación españoles ven en estos recursos mass-media una forma de superar esta realidad. No en vano el libro de texto es el recurso peor valorado en los futuros docentes españoles, mientras que en el caso inglés no está tan mal valorado (casi 3,5 sobre 5). Unos resultados que muestran también una realidad distinta de los libros de texto ingleses, con actividades más procedimentales y más motivacionales frente a los españoles (Gómez y Chapman, 2017; MartínezHita y Gómez, 2018).

Esta explicación se refuerza con la valoración de los cómics y los videojuegos. Aunque ocupan un lugar inferior en el caso de España, ambos superan la valoración de 3 sobre 5 , mientras que en el caso inglés tienen 
una valoración muy baja. En España los estudios Cózar y Sáez (2016), Jiménez y Cuenca (2015 y 2017) o Sáez y Cózar (2017) muestran cómo el uso de videojuegos puede mejorar el proceso de enseñanza-aprendizaje de las ciencias sociales en torno a dos factores: motivación y aprendizaje experiencial. Pero estos resultados se muestran como contraposición a la clase tradicional y expositiva hegemónica en las aulas españolas. En Inglaterra, donde no existe esa supremacía de la clase tradicional, no valoran a estos recursos como válidos para la enseñanza de la Historia, y sí valoran más positivamente aquellos que permiten desarrollar los procedimientos del historiador.

Estos estudios de diagnóstico nos permiten dimensionar la necesidad de formar docentes altamente cualificados para mejorar los procesos de enseñanza-aprendizaje en educación obligatoria. Un tema candente a nivel internacional (Barnes, Fives y Dacey, 2017; König \& Blömeke, 2012). El análisis de los conocimientos de los docentes en formación se considera crucial para orientar los programas de formación inicial del profesorado (Darling-Hammond, 2006). Existe abundante bibliografía que ha analizado las creencias y percepciones de los docentes en formación para comprender cómo se pueden utilizar estos constructos para mejorar su formación (Fives \& Buehl, 2012; Pajares, 1992).

Con estos resultados podemos incidir en la necesidad de plantear en la formación del profesorado estrategias que permitan aunar en las aulas los aspectos motivacionales y el aprendizaje experiencial en los recursos relacionados con las TIC y los mass-media. Mientras que el uso de los mismos no se utilice para incentivar los procedimientos del historiador, para plantear interrogantes en el aula como proponían Cuenca, Martín y Estepa (2011), solo se podrá vislumbrar en ellos una función lúdica y motivacional, realidad que está presente en las aulas de ciencias sociales desde hace al menos veinte años (Valero y Vera, 1998). Es necesario sobrepasar esa percepción en los futuros docentes, a través de una intervención en su formación, que les permita usar estos recursos en el aula de Historia. Todas estas herramientas por sí mismas, si no se utilizan de forma adecuada, no originan mejoras en la educación histórica (Miralles, 2011). Si la metodología utilizada sigue siendo tradicional, las TIC no servirán de mucho. El uso de estos medios debe servir para fomentar el pensamiento autónomo, creativo y crítico. Internet debe ser un instrumento para cuestionar el conocimiento, para aprender a pensar históricamente y es necesario enseñar a hacerlo. El mito de los nativos digitales, con una mala interpretación del ensayo de Prensky, es falso. La digital, como cualquier alfabetización, necesita ser enseñada y aprendida. 


\section{NOTAS}

1 El presente trabajo es fruto de los proyectos de investigación «La evaluación de las competencias y el desarrollo de capacidades cognitivas sobre historia en Educación Secundaria Obligatoria»(EDU2015-65621-C3-2-R), financiado por el Ministerio de Economía y Competitividad y cofinanciado por los fondos FEDER; y "Competencias docentes y métodos activos de aprendizaje. Una investigación evaluativa con el profesorado en formación de ciencias sociales» (20638/JLI/18), financiado por la Fundación Séneca. Agencia de Ciencia y Tecnología de la Región de Murcia.

2 Queremos agradecer al profesor Arthur Chapman la ayuda prestada en la recogida de información del cuestionario en Inglaterra, ya que nos ayudó a ponernos en contacto con todas las universidades inglesas de las que se ha recogido información. 


\section{REFERENCIAS BIBLIOGRÁFICAS}

Acosta, L.M. (2010). La enseñanzaaprendizaje de la historia en bachillerato y las TIC: la introducción de la estrategia Webquest. Clio. History and History Teaching, 36.

Ayén, F. (2017). ¿Qué es la gamificación y el ABJ? Íber. Didáctica de las Ciencias Sociales, Geografía e Historia, 86, 7-15.

Barnes, N., Fives, H., \& Dacey, C. (2017). U.S. teachers' conceptions of the purposes of assessment. Teaching and Teacher Education, 65, 107-116.

Buendía, L., Colás, P. y Hernández-Pina, F. (1998). Métodos de investigación en psicopedagogía. Madrid: McGraw-Hill.

Cabero-Almenara, J. y Guerra-Liaño, S. (2011). La alfabetización y formación en medios de comunicación en la formación inicial del profesorado. Educación XX1, 14(1), 89-115.

Colomer, J.C. (2016). Historypin: una app para el trabajo del pensamiento histórico en Didáctica de las Ciencias Sociales. En IV Congreso Internacional de Investigación en Innovación Educativa (pp. 171-176). Murcia: Universidad de Murcia.

Contreras-Espinosa, R., Eguía-Gómez, J. L., y Solano Albaje, L. (2011). Videojuegos como un entorno de aprendizaje. El Caso de "Monturiol el joc». Revista ICONO14. Revista científica de Comunicación $y$ Tecnologias emergentes, 9(2), 249-261.

Cooper, H. \& Chapman, A. (Ed.) (2009). Constructing History. London, UK: Sage.

Cózar, R. \& Sáez, J.M. (2016). Gamebased learning and gamification in initial teacher training in the social sciences: an experiment with MinecraftEdu. International Journal of Educational Technology in Higher Education, 13(2), 1-11.

Cózar, R. y Sáez, J. M. (2017). Realidad aumentada, proyectos en el aula de primaria: experiencias y casos en Ciencias Sociales. EDMETIC, Revista de Educación Mediática y TIC, 6(1), 165-180.

Cózar, R., De Moya, M.V., Hernández, J.A., y Hernández, J.R. (2015). Tecnologías emergentes para la enseñanza de las Ciencias Sociales. Una experiencia con el uso de Realidad Aumentada en la formación inicial de maestros. Digital Education Review, 27, 138-153.

Cózar, R., Zagalaz, J., y Sáez, J.M. (2015). Creando contenidos curriculares digitales de Ciencias Sociales para Educación Primaria. Una experiencia TPACK para futuros docentes. Educatio Siglo XXI, 33, 147-168. doi: https://doi.org/10.6018/j/240921

Cuenca, J.M., Martín, M., y Estepa, J. (2011). Historia y videojuegos. Una propuesta de trabajo para el aula de 1 . $^{\circ}$ de ESO. Íber. Didáctica de las Ciencias Sociales, Geografía e Historia, 69, 6473.

Cuesta, R. (1998). Clío en las aulas. La enseñanza de la Historia en España entre reformas, ilusiones y rutinas. Madrid: Akal.

Darling-Hammond, L. (2006). Assessing teacher education. The usefulness of multiple measures for assessing program outcomes. Journal of Teacher Education, 57(2), 120-138

De Haro, J.J. (2009). Algunas experiencias de innovación educativa. ARBOR. Ciencia, pensamiento $y$ cultura, CLXXXV, 71-92. 
De la Torre, J.L. (2005). Las nuevas tecnologías en las clases de ciencias sociales del siglo XXI. Quaderns Digitals, 37. Recuperado de https://bit. ly/2WH06PJ

Del Moral, M.E., Villalustre, L., Yuste, R., y Esnaola, G. (2012). Evaluación y diseño de videojuegos: generando objetos de aprendizaje en comunidades de práctica. RED. Revista de Educación a Distancia, 33.

Domínguez, J. (2015). Evaluación del pensamiento histórico y conceptos metodológicos de la historia. En J. Domínguez, Pensamiento histórico y evaluación de competencias (pp. 3564). Barcelona: Graó.

Fives, H., \& Buehl, M.M. (2014). Exploring differences in practicing teachers' valuing of pedagogical knowledge based on teaching ability beliefs. Journal of Teacher Education, 65(5), 435-448. http://dx.doi. org/10.1177/0022487114541813

Gómez, C. J., Miralles, P., y Chapman, A. (2017). Los procedimientos de evaluación en la clase de Historia. Un análisis comparativo a través de las opiniones de los docentes en formación en España e Inglaterra. Revista Electrónica Interuniversitaria de Formación del Profesorado, 20(2), 45-61.

Gómez, C.J., Rodríguez, R.A. y Mirete, A.B. (2018). Percepción de la enseñanza de la historia y concepciones epistemológicas. Una investigación con futuros maestros. Revista Complutense de Educación, 29(1), 237-250.

Gómez, I. (2015). El modelo TPACK en los estudios de grado para la formación inicial del profesorado en TIC. Didáctica Geográfica, 16, 185-201.

Gómez, I.M. (2016). La adquisición del conocimiento base del docente en ciencias sociales a través del modelo de enseñanza y aprendizaje TPACK en la formación inicial del profesorado con tecnología. Revista Internacional de Investigación en Didáctica de las Humanidades y Ciencias, 3, 123-138.

González-Fernández, N., GozálvezPérez, V., y Ramírez-García, A. (2015). La competencia mediática en el profesorado no universitario. Diagnóstico y propuestas formativas. Revista de Educación, 367, 117-146. doi: 10.4438/1988-592X-RE-2015-367284

Gorgues, R. y Goberna J.J. (1998). El cine en la clase de Historia. Comunicar, 11, 87-93.

Gros, B. (2008) (Coord.). Videojuegos y aprendizaje. Barcelona: Graó.

Guevara, J.M. y Colomer, J.C. (2017). Minecraft y Eduloc en historia y geografía. Íber. Didáctica de las Ciencias Sociales, Geografía e Historia, 86, 16-23.

Hernàndez Cardona, F.X. (2011). La iconografía en la didáctica de las ciencias sociales. Íber. Didáctica de las Ciencias Sociales, Geografía e Historia, 68, 7-16.

Hernández, F. y Maquilón, J.J. (2010). Introducción a los diseños de investigación educativa. En S. Nieto (Ed.), Principios, métodos y técnicas esenciales para la investigación educativa (pp. 109-126). Madrid: Dykinson.

Ibáñez, A., Vicent, N. y Asensio, M. (2012). Aprendizaje informal, patrimonio y dispositivos móviles. Evaluación de una experiencia en educación secundaria. Didáctica de las Ciencias Experimentales y Sociales 26, 3-18. 
Ibáñez, A., Vicent, N., Asensio, M., Cuenca, J. M. \& Fontal, O. (2014). Learning in archaeological sites with mobile devices. Munibe, 65, 313-321.

Iturriaga, D. (2015). Enseñar historia haciendo visible lo invisible a través de los videojuegos de historia en Secundaria. En A.M. Hernández, C.R, García, y J.L. de la Montaña (Eds.), Una enseñanza de las ciencias sociales para el futuro: recursos para trabajar la invisibilidad de las personas, lugares y temáticas (pp. 217-222). Cáceres: Universidad de Extremadura.

Iturriaga, D. y Medel, I. (2017). La historia a través de los videojuegos. Íber. Didáctica de las Ciencias Sociales, Geografía e Historia, 86, 30-36.

Jiménez, J.F. \& Rodríguez, G. (2015). Medieval soundspace in the new digital leisure time media. Imago Temporis, 9, 307-327.

Jiménez, R. y Cuenca J.M. (2015). El uso didáctico de los videojuegos. Concepciones e ideas de los futuros docentes de ciencias sociales. Clío. History and History Teaching, 41.

Jiménez, R. y Cuenca, J. M. (2017). Libertus. Íber. Didáctica de las Ciencias Sociales, Geografía e Historia, 86, 4144.

König, J., \& Blömeke, S. (2012). Future Teachersö General Pedagogical Knowledge from Comparative Perspective. Does School Experience Matter? ZDM - The International Journal on Mathematics Education, 44, 341-354.

Lee, P. (2005). Putting Principles into Practice: Understanding History. En M.S. Donovan \& J.D. Bransford (Eds.), How students learn: History in the classroom (pp. 29-78). Washington, DC, USA: National Academies Press.
Lee, P. \& Ashby, R. (2000). Progression in Historical Understanding among Students Ages 7-14. En P.N. Stearns, P. Seixas, \& S. Wineburg (Eds.), Knowing, Teaching, and Learning History: National and International Perspectives (pp. 199-222). New York, NY, USA: University Press.

Lee, P., Dickinson, A. y Ashby, R. (2004). Las ideas de los niños sobre la historia. En M. Carretero y J.F. Voss (Coords.), Aprender y pensar la historia (pp. 217-248). Buenos Aires, Argentina: Amorrortu.

López-García, A. y Miralles, P. (2018). La realidad aumentada en la formación del profesorado. Una experiencia en las prácticas del Máster de Profesorado de Enseñanza Secundaria. Campus Virtuales, 7(2), 39-46.

López-Menchero, V.M. y Ramiro, R. (2015). La arqueología virtual como recurso para la comprensión del paisaje cultural. Íber. Didáctica de las Ciencias Sociales, Geografía e Historia, $81,41-44$

Marqués, P. (2013). Impacto de las TIC en la educación: funciones y limitaciones. 3 c TIC: cuadernos de desarrollo aplicados a las TIC, 2(1), 2-15.

Martín, C. (2011). Tecnologías digitales interactivas y didáctica de las ciencias. Íber. Didáctica de las Ciencias Sociales, Geografía e Historia, 68, 33-39.

Martínez-Hita, M. y Gómez, C.J. (2018). Nivel cognitivo y competencias de pensamiento histórico en los libros de texto de Historia de España e Inglaterra. Un estudio comparativo. Revista de Educación, 379, 149-165.

Martínez, A.M. (2017). Game in Florence. Íber. Didáctica de las Ciencias Sociales, Geografía e Historia, 86, 37-40. 
Martínez, N., Miralles, P., y Navarro, E. (2009). Análisis de cómo los docentes plantean el inicio de las clases, desde la perspectiva del alumnado. Revista de Educación, 349, 413-433.

Martínez, N., Souto, X.M., y Beltrán, J. (2006). Los profesores de historia y la enseñanza de la historia en España. Una investigación a partir de los recuerdos de los alumnos. Enseñanza de las ciencias sociales: revista de investigación, 5, 55-71.

Maté, I. (2017). Juegos de rol. Pautas para su uso en clase. Íber. Didáctica de las Ciencias Sociales, Geografía e Historia, 86, 24-29.

Merchán, F.J. (2007). El papel de los alumnos en la clase de historia como agentes de la práctica de la enseñanza. Didáctica de las Ciencias Experimentales y Sociales, 21, 33-51.

Miralles, P. (2011). La historia y el uso de las nuevas tecnologías de la información y la comunicación. En Enseñanza y aprendizaje de la Historia en la Educación Básica (pp. 126-142). México D.F., México: Secretaría de Educación Pública y Universidad Pedagógica Nacional de México.

Miralles, P., Gómez, C.J. y Arias, L. (2013). La enseñanza de las ciencias sociales y el tratamiento de la información. Una experiencia con el uso de webquests en la formación del profesorado de educación primaria. Revista de Universidad y Sociedad del Conocimiento (RUSC), 10(2), 98-111.

Miralles, P., Gómez, C.J. y Rodríguez, R.A. (2017). Patrimonio, competencias históricas y metodologías activas de aprendizaje. Un análisis de las opiniones de los docentes en formación en España e Inglaterra. Estudios Pedagógicos, XLIII(4), 161-183.
Ortega-Carrillo, J.A. y Pérez-García, A. (2013). El cine digital en la formación inicial del profesorado: una experiencia innovadora realizada en la Universidad de Granada. Educación $X X 1,16(2), 297-320$. doi: 10.5944/ educxx1.16.2.2644

Osset, J. (2004). Las nuevas tecnologías en ciencias sociales. Un viaje hacia el aula virtual. Íber. Didáctica de las Ciencias Sociales, Geografía e Historia, 4, 57-67.

Pajares, M.F. (1992). Teachers' beliefs and educational research: Cleaning up a messy construct. Review of Educational Research, 62(3), 307-332. http:// dx.doi. org/10.3102/00346543062003307

Ramírez-García, A. y GonzálezFernández, N. (2016). Competencia mediática del profesorado y del alumnado de educación obligatoria en España/Media Competence of Teachers and Students of Compulsory Education in Spain. Comunicar, 24(49), 49-58.

Rivero, P. (2010). El aprendizaje multimedia de la Historia: valoración de una experiencia de aula. En R. López-Facal, L. Velasco, V. Santidrián y X. Armas (Eds.), Pensar históricamente en tiempos de globalización. Congreso internacional sobre la enseñanza de la Historia (pp. 190-199). Santiago de Compostela: Universidad de Santiago de Compostela.

Rivero, P. (2011). Un estudio sobre la efectividad de la multimedia expositiva para el aprendizaje de la Historia. Enseñanza de las Ciencias Sociales. Revista de investigación, 10, 45-50.

Rodríguez, M.A. y Gutiérrez, D. (2016). Innovación en el aula de ciencias sociales a través de los videojuegos. Revista Iberoamericana de Educación, 72 (2), 181-200. 
Romero-Martín, R., Castejón-Oliva, F. J., López-Pastor, V.M. y Fraile-Aranda, A. (2017). Evaluación formativa, competencias comunicativas y TIC en la formación del profesorado. Comunicar. Revista Científica de Comunicación y Educación, 25(52), 73-82.

Sáez, J.M. y Cózar, R. (2017). Programación visual por bloques en Educación Primaria: aprendiendo y creando contenidos en ciencias sociales. Revista Complutense de Educación, 28(2), 409-426.

Sánchez, Alarcón, I. (1999). El cine, instrumento para el estudio y la enseñanza de la Historia. Comunicar 13, 159-164.
Sobrino, D. (2013). El trabajo con blogs en ciencias sociales, geografía e historia. Clio. History and History Teaching, 39.

Téllez, D. y Iturriaga, D. (2014). Videojuegos y aprendizaje de la historia: la saga Assasin's Creed. Contextos educativos, 17, 145-155.

Valero Palomo, M. P. y Vera Aranda, A.L. (1998). Medios de comunicación en clase de Geografía e Historia Comunicar, 11, 130-136.

Vicent, N., Ibáñez, A. y Asensio, M. (2015). Evaluación de programas de educación patrimonial de base tecnológica. Virtual Archaeology Review, 13(6), 18-25. 


\section{PERFIL ACADÉMICO Y PROFESIONAL DE LOS AUTORES}

Pedro Miralles Martínez. Profesor Titular de Didáctica de las Ciencias Sociales en la Universidad de Murcia. Investigador principal del grupo Didáctica de las Ciencias Sociales. Doctor en Geografía e Historia. Ha sido profesor de EGB, profesor y catedrático de Geografía e Historia de Enseñanza Secundaria y asesor de formación permanente del profesorado. Es autor de más de dos centenares de publicaciones académicas entre artículos, libros, capítulos de libros y participación de congresos nacionales e internacionales. Ha dirigido varios proyectos de investigación e innovación sobre didáctica de las ciencias sociales, enseñanza-aprendizaje de la historia y la evaluación de los contenidos geográficos e históricos.

Cosme J. Gómez Carrasco. Profesor Titular de Didáctica de las Ciencias Sociales de la Universidad de Murcia. Doctor en Historia por la UCLM, ha estado vinculado con anterioridad a la EHESS (París) y la Universidad Carlos III de Madrid como investigador postdoctoral. También ha sido profesor invitado en Centre de Recherches Historiques (EHESS, París) y en la University College of London-Institute of Education. Autor de más de un centenar de aportaciones entre artículos, libros y capítulos de libro, ha participado en una quincena de proyectos de investigación sobre Historia Moderna y Didáctica de las Ciencias Sociales. Sus líneas de investigación actuales son: pensamiento histórico, formación del profesorado de Ciencias Sociales, libros de texto de Historia, y evaluación.

José Monteagudo Fernández. Profesor Contratado Doctor de Didáctica de las Ciencias Sociales de la Universidad de Murcia. Ha sido anteriormente Profesor Asociado en la misma universidad y profesor de Geografía e Historia de Enseñanza Secundaria. Ha participado como investigador o colaborador en varios proyectos de investigación financiados en convocatorias públicas. Autor de varias aportaciones científicas, sus líneas de investigación son la evaluación del proceso de enseñanza-aprendizaje y la didáctica del patrimonio en las etapas de Educación Primaria y Secundaria.

Dirección de los autores: Universidad de Murcia

Facultad de Educación

Dpto. de Didáctica de las CC Matemáticas y

Sociales

Campus de Espinardo

30100 Murcia, España

E-mail: pedromir@um.es

cjgomez@um.es

jose.monteagudo@um.es

Fecha Recepción del Artículo: 20. Febrero. 2018

Fecha Modificación del Artículo: 20. Septiembre. 2018

Fecha Aceptación del Artículo: 01. Octubre. 2018

Fecha Revisión para Publicación: 01. Febrero. 2019 
